\title{
Contents of Volume 51
}

Isabelle von Bueltzingsloewen (Lyon), Naissance de la clinique allemande (Birth of the German Clinic)

Robert W. Carrubba (Oshkosh/WI), Pastor H.G. Weland's Latin Elegy for Engelbert Kaempfer

Dietrich von Engelhardt (Lübeck), Paracelsus im Urteil des 18. Jahrhunderts (Paracelsus in the Opinion of the 18th Century)

Stefan Hächler (Bern), Zur Gründungsgeschichte der Schweizerischen Krebsliga (Foundation and Development of the Swiss Leage Against Cancer)

Helmut Knolle (Bern), Zur historisch-geographischen Epidemiologie der Poliomyelitis (Historico-geographical Epidemiology of Poliomyelitis)

Micheline Louis-Courvoisier (Genève), L'Hôpital général de Genève de 1780-1798: quelque indice de médicalisation (The General Hospital of Geneva 1780-1798: Indications of Medicalization)

Josef N. Neumann (Halle/S), Die wissenschaftstheoretische Begründung der Medizin als Naturwissenschaft bei Hermann von Helmholtz (1821-1894) (Helmholtz' Theoretical Foundation of Medicine as a Science)

Ortrun Riha (Göttingen), Kommunikationsprobleme in der medizinischen Mittelalterforschung (Communication Problems in Research of Medieval History of Medicine)

Heinrich Schipperges (Heidelberg), Vom Geist der Natur bei Paracelsus (Paracelsus and the Spirit of Nature)

Josef M. Sснмidt (München), Die Entwicklung der Homöopathie in den Vereinigten Staaten (The Development of Homeopathy in the United States)

Marie-France Vouilloz Burnier (Vevey), Un manuel empirique pour la formation des sages-femmes valaisannes (An Empirical Manual for the Training of Midwives in the Canton of Valais)

Karl Wiederkehr (Hamburg), Wilhelm Weber und Maxwells elektromagnetische Lichttheorie (Wilhelm Weber and Maxwell's Electro-magnetic Theory of Light) 


\section{Short Communications}

Pere Domingo and Nicolau Barquet (Barcelona), The First Epidemic of Cerebrospinal Meningitis

Kazimierz Karbowski et Filippo Donati (Bern), Sur la dédicace du «Sermo inauguralis» (1766) de Samuel-Auguste Tissot (On the Dedication of Samuel-Auguste Tissot's "Sermo inauguralis» of 1766)

Guy Meynell (Dover/GB), Surgical Teaching at the Jardin des Plantes During the Seventeenth Century

Christoph Mörgeli (Zürich), Ein Dokument. Manuskript eines Nachrufs von 1873 auf den Schweizer Medizinhistoriker Conrad Meyer-Ahrens ( $A$ Document. Manuscript of an Obituary of 1873 for the Swiss Historian of Medicine, Conrad Meyer-Ahrens)

Obituaries

News AND Activities

Prof. Dr. Jean Starobinski

Prof. Dr. Marcel H. Bickel

Die Jahrestagung der Schweizerischen Gesellschaft für Geschichte der Medizin und der Naturwissenschaften

Gesnerus Register

IInd International Symposium Galdeano

Zweite Jahrestagung der Deutschen Gesellschaft für Geschichte und Theorie der Biologie in Konstanz

Histoire et actualité de la cosmologie

Création d'un Institut universitaire romand d'histoire de la médecine et de la santé

Centenaire de la découverte du bacille de la peste

7. Kongress der European Association of Museums of the History of Medical Sciences (EAMHMS) in Zürich

A new Post: Laureate of the History of Anesthesia

Arbeitskreis Alte Medizin

Books

Essay Reviews

Henri F. Ellenberger, Histoire de la découverte de l'inconscient; Mark S. Micale, éd., Beyond the Unconscious. Essays of Henri F. Ellenberger in the History of Psychiatry (Vidal)

Danielle Gourevitch (ed.), Maladie et maladies, histoire et conceptualisation (Rudolph)

Monographs

Wilfred Niels Arnold, Vincent van Gogh, ein Leben zwischen Kreativität und Krankheit (Müller-Thalheim)

Atti del convegno celebrativo di Johann Georg Wirsung nel quarto centenario della nascità (Bonuzzi)

Josep Lluís Barona, Introducció a la medicina (Keller-Schnider) 
Marco Beretta, A History of Non-Printed Science. A select catalogue of the Waller Collection (Seger)

Claudia Bergemann, Mitgliederverzeichnis der Kaiser-Wilhelm-Gesellschaft zur Förderung der Wissenschaften (Koelbing)

Georg Cantor, Briefe. Hrsg. von Herbert Meschkowski und Winfried Nilson (Burckhardt)

Samuel K. Cohn, Jr., The Cult of Remembrance and the Black Death (Seiler)

Lothar Dittrich, Sigrid Dittrich, Ingrid Faust, Das Bild der Giraffe (Ziswiler)

Barbara Duden, Body History - Körpergeschichte. A repertory - ein Repertorium (Müller-Landgraf)

Richard J.Durling, A Dictionary of Medical Terms in Galen (Burkert)

Heidemarie Emisch, Ludwig Edinger - Hirnanatomie und Psychologie (Graf-Nold)

Gerhard Fichtner (Hrsg.), Sigmund Freud-Ludwig Binswanger : Briefwechsel 1908-1938 (Walser)

Friedrich Hermann Fischer, Der Missionsarzt Rudolf Fisch und die Anfänge medizinischer Arbeit der Basler Mission an der Goldküste (Ghana) (Koelbing)

Galen, On semen/De semine (Zimmermann)

Werner A. Gehret, Gehirn und Professoren. $80+$ ein Semester an der Universität Zürich (Karbowski)

Leonie de Goei and Joost Vijselaar (eds.), Proceedings of the 1st European Congress on the History of Psychiatry and Mental Health Care, 's-Hertogenbosch, The Netherlands, 24-26 October 1990 (Ernst)

Ove Hagelin, Rare and important medical books in the Library of the Karolinska Institute (Seger)

Anne Harrington (ed.), So human a brain. Knowledge and values in the neurosciences (Hagner)

Wolfgang-Hagen Hein, Wolf-Dieter Müller-Jahncke, Kostbarkeiten aus dem Deutschen Apotheken-Museum Heidelberg (Ledermann)

Eckart Henning und Marion Kazemi, Chronik der Max-Planck-Gesellschaft zur Förderung der Wissenschaften unter der Präsidentschaft Otto Hahns (1946-1960) (Glaus)

Ekkehard Hlawitschka, «wazzer der tugent, trank der jugent». Text- und überlieferungsgeschichtliche Untersuchungen zum Salbeitraktat (Wäckerlin-Swiagenin)

Urs Michael Humbel, Zur Geschichte der Antimykotika in der zweiten Hälfte des 19. Jahrhunderts. (Elsner)

Saul Jarcho, Quinine's predecessor. Francesco Torti and the early history of Cinchona. (Bickel)

Dieter Jetter, Geschichte der Medizin: Einführung in die Entwicklung der Heilkunde aller Länder und Zeiten (Raggenbass)

Dieter Jetter, Geschichte der Medizin: Einführung in die Entwicklung der Heilkunde aller Länder und Zeiten (Ritzmann)

Carl Friedrich Kielmeyer, Über die Verhältnisse der organischen Kräfte unter einander in der Reihe der verschiedenen Organisationen, die Gesetze und Folgen dieser Verhältnisse (Ziswiler)

Volker Langholf, Medical theories in Hippocrates. Early texts and the «Epidemics» (Hoessly)

Larissa Leibrock-Plehn, Hexenkräuter oder Arznei: die Abtreibungsmittel im 16. und 17. Jahrhundert (Fehlmann)

Leopoldina-Meeting zur Edition naturwissenschaftlicher Texte der Goethezeit (Glaus) 
Ludwig Limmer, Günter K. Krieglstein, Augenheilkunde im Rom der frühen Kaiserzeit: A.C. Celsus, De medicina libri octo (Koelbing)

Dieter Lohmeier und Bernhardt Schell (Hrsg.), Einstein, Anschütz und der Kieler Kreiselkompass (Funk)

William P. Longmire, Jr., Alfred Blalock - his life and times (Naef)

Andreas-Holger Maehle, Kritik und Verteidigung des Tierversuchs (Lindenmann)

F. E. R. de Maar, Een gebit zonder eind. Grepen uit 50 eeuwen geschiedenis van de mondhygiëne (Rosenberg)

Pirmin Meier, Paracelsus, Arzt und Prophet (Koelbing)

Christian Müller, Vom Tollhaus zum Psychozentrum (Ernst)

Ingo Wilhelm Müller, Iatromechanische Theorie und ärztliche Praxis im Vergleich zur galenistischen Medizin (Friedrich Hoffmann-Pieter van Foreest, Jan van Heurne (Boschung)

Thomas Nickol, Das wissenschaftiche Werk des Arztes und Zahnarztes Carl Röse (1864-1947) (Sigron)

Wolfgang Michael Pahl, Altägyptische Schädelchirurgie. Untersuchungen zur Differentialdiagnose von Trepanationsdefekten und zur Frage der Realisierung entsprechender Eingriffe in einem elaborierten prähippokratischen Medizinsystem (Böni)

John Parascandola, The Development of American Pharmacology. John J. Abel and the Shaping of a Discipline (Bickel)

Véronique Pasche, "Pour le salut de mon âme». Les Lausannois face à la mort (XIVe siècle) (Seiler)

La Philosophie du remède. Sous la direction de Jean-Claude Beaune. (Actes du colloque 1992) (Lindenmann)

Ingrid Pieroth, Penicillinherstellung (Bickel)

K.A. Portele, Die Sammlung mazerierter Skelette und Knochen des Pathologisch-anatomischen Bundesmuseums in Wien, Teil II (postcraniale Knochen) (Ulrich)

Paul Ridder, Chirurgie und Anästhesie. Vom Handwerk zur Wissenschaft (Naef)

John M. Riddle, Quid pro quo: Studies in the history of drugs (Mudry)

Owsei Temkin, Hippocrates in a world of pagans and Christians (Koelbing)

Gordon Uhlmann und Ursula Weisser (Hrsg.), Krankenhausalltag seit den Zeiten der Cholera (Ritzmann)

Silvano Villa, Beiträge von Apothekern zur Bakteriologie, Desinfektion und Hygiene in der zweiten Hälfte des 19. Jahrhunderts (Graevenitz)

Rudolf Virchow, Sämtliche Werke. Hrsg. von Christian Andree. Abt. I [Bde. 127] Medizin, Band 4: Beiträge zur wissenschaftlichen Medizin aus den Jahren 1846-1850 (Koelbing)

Rudolf Virchow, Sämtliche Werke. Hrsg. von Christian Andree. Abt. II [ Bde 28-41] Politik (Mörgeli)

Peter Voswinckel, Der schwarze Urin (Stettler)

Magda Whitrow, Julius Wagner-Jauregg (1857-1940) (Müller) 